\title{
HISTORY
}

OF

\section{A CASE OF STAMMERING,}

\author{
SUCCESSFULLY TREATED BY THE LONG CONTINUED \\ USE OF CATHARTICS.
}

By JOHN BOSTOCK, M.D. F.R.S.

Read March 23, 1830.

IMPEDIMENTS of speech are usually regarded as originating either in a physical defect of the organs which are exercised in the production of articulate sounds, or as proceeding from some cause more of a mental nature, as habit, imitation, or the like. The modes of treatment that have been proposed, as far as we are made acquainted with them, are accordingly adapted to one or other of these supposed causes, and consist either in certain methods of managing the muscles that are concerned in speech, or in counteracting those circumstances which may be supposed to induce the habit, or the tendency to imitation. A case has fallen under my observation, which has 
led me to take a different view of the subject; and as the treatment has been, upon the whole, successful, I presume that a short account of it will be acceptable to the society.

A boy of a robust form and florid aspect, of a healthy constitution, and of more than ordinary activity both of mind and body, when between two and three years old, and after having acquired considerable readiness in speaking, was suddenly affected with so great a degree of stammering as to be almost incapable of uttering a single syllable. Two eminent physicians were consulted: they confessed their inability to propose any specific plan of treatment which might afford a prospect of success, but in consequence of a somewhat plethoric state of the child, they advised that a strong purgative should be given. The effect of the medicine appeared so favourable, that it was repeated three or four times, and each time with such decided benefit, as to leave no doubt on this point in the minds either of the parents or the practitioners. The complaint, however, shortly recurred, was again attacked with the same remedy, and was again subdued. After this plan had been continued for some time, it was conceived that, in addition to the purgative system, the effect of which, although so salutary, was temporary, further advantage might be obtained by adopting a system of diet which should permanently reduce the plethoric habit, and obviate the 
necessity for the continual repetition of the purgatives. This was accordingly done, and was rigidly adhered to for several years. Animal food was totally abstained from, and even vegetables were taken in as sparing a quantity as was consistent with the support of the system. The effect of this regimen was sufficiently apparent in the altered aspect of the child, who became much less plump and florid, but still retained a due share of vigour and activity, and was fully adequate to enter into all the sports and exercises suited to his age. I had frequent opportunities of witnessing the result of this plan; and I may venture to assert, that it is impossible to have stronger evidence of the beneficial operation of any medical treatment, than is presented by the case in question.

By a steady adherence to this discipline for about eight years, the complaint was kept at bay; but whenever any relaxation in the diet took place, or when the purgatives were omitted or too long delayed, symptoms of the impediment immediately appeared. At length, when about twelve years of age, the tendency seemed so far subdued, that a relaxation of the restrictions was not followed by the usual unfavourable consequences, and the boy being then at a public school, it was not so easy to maintain the former discipline. For some time no bad effects ensued, but at length the complaint recurred, and was unusually obstinate, so as to require a long and 
severe course of purgatives, which, however, was finally successful.

During the last two years the tendency has occasionally manifested itself, but it has always been easily removed by a moderate use of purgatives, and by a temperate, although not a rigidly abstemious diet. The boy, who is now in his fifteenth year, may be said to be free from the complaint. No one but those who are aware of the circumstances of the case, and were on the watch to detect even a slight defect, would notice any thing peculiar in his mode of speaking. He even possesses a considerable rapidity and volubility of enunciation; and as a proof of this I may state, that I was lately present at a juvenile exhibition, when he bore a conspicuous part in a comic dialogue, in which he displayed a complete command over the organs of speech. In this respect, he may be favourably contrasted with many of those individuals who have been under the care of the masters who profess to remove these impediments. It would be unjust not to admit, that they occasionally produce very beneficial effects on those committed to their care, but I think it may be asserted, that in all these cases, there is a certain peculiarity in the mode of speaking, which although much preferable to decided stammering, indicates that the difficulty is rather evaded than obviated. 
With respect to the purgatives employed in this case, it appeared to be of little importance which were used, provided the bowels were very completely evacuated. What was the most frequently employed was a full dose of calomel and jalap, succeeded by Epsom salts. Whenever the examination was inade, it was found that the fæces were in a morbid state; and while the child was young, and the examination could be easily made, the necessity for continuing the medicine was judged of as much by the appearance of the fæces as by the state of the symptoms. It happened, on two or three occasions, that a degree of salivation was unintentionally excited, but it was not easy to determine whether this circumstance was productive of any advantage, as the relaxation of the bowels was contemporary and proportional.

How far we may be allowed to draw a general inference from a single case, I will not venture to decide; but I may be allowed to say, that a trial should be made of a plan of treatment which is productive of no inconvenience, which involves no expense, and does not interfere with education, or with the ordinary habits of life. It may be difficult to determine how far such a process should be recommended to adults. Much must depend upon age, constitution, temperament, \&c., but I should suppose that few individuals would object to submit to a trial, although the hope of success may not be considerable. 
As I propose this communication to be of a practical nature, I abstain from entering into any pathological observations on the nature and cause of the affection, further than to remark, that the complaint appears to consist essentially in a loss of power over certain voluntary muscles, and that as the muscles themselves do not seem to be affected, it ought probably to be referred to the class of nervous diseases, and may be regarded as analogous to chorea, differing from it principally in its seat, and in its being confined to one set of muscles, while chorea affects a much greater number of parts, and produces a proportionally greater disturbance of the constitution and functions.

Upper Bedford Place, Feb. 3, 1830. 